\title{
Sakurai's object - stellar evolution in real time
}

\author{
Martin Asplund \\ Nordita, Blegdamsvej 17, DK-2100 København Ø, Denmark
}

\begin{abstract}
The born-again giant Sakurai's object is currently experiencing its second stage as an AGB-star. Furthermore, Sakurai's object has shown an unprecedented rapid stellar evolution since discovery in 1996, both in terms of a continued cooling of the photosphere and spectacular changes in chemical composition on a time-scale of a mere few months. The surface cooling and abundance alterations of $\mathrm{H}, \mathrm{Li}$ and the $s$-elements are the direct consequences of the expansion, mixing and nucleosynthesis which has ensued as a result of a final He-shell flash occurring while the star was descending the white dwarf cooling track. Sakurai's object shows striking similarities with the $\mathrm{R} \mathrm{CrB}$ stars in both chemical composition and visual variability.
\end{abstract}

\section{Introduction}

The incongruity of the time-scales of stellar and human life-times has throughout much of human evolution resulted in the impression that stars are immutable. Today we know that stars are born, live and die, though the time-scale for this evolution is typically million or billions of years. Our understanding of stellar evolution thus predominantly comes from observing many stars in different evolutionary stages and relying on theoretical modelling. Witnessing stellar evolution in real time is therefore extraordinarily rare. Supernovae and novae provide of course notable exceptions, though such cataclysmic events only represent the ultimate end as normal stars. "Born-again" giants, however, offer a unique opportunity to study stellar evolution on a time-scale more appropriate to the life-time of astronomers, which gives important direct clues to how stellar evolution and nucleosynthesis proceeds during late stages of stellar evolution.

During the descent of the white dwarf cooling track a final He-shell flash (thermal pulse) may occur, which balloons the star back to supergiant dimensions due to the released energy from the once-again initiated nuclear burning. The star thus makes a second appearance as an AGB-star: a born-again giant. Though rarely observed, such a scenario is not unlikely for intermediate- and low-mass stars: theoretical estimates suggest that about 10-20\% of such stars experience a phase as born-again giants. The life-time as a rejuvenated giant is, however, short - typically only 100-1000 years - before the star re-traces its previous evolution towards the planetary nebulae phase and eventually the white dwarf domain, this time with no turning back.

Only a few candidates for having been born-again giants are known. Best known is perhaps FGSge which has evolved from being an $\mathrm{O}$ star a century ago 
to now being a late-type giant. V605 Aql and a few central stars of PNe may also have experienced a similar final He-shell flash, and the R CrB stars may be descendants of such events (Asplund et al. 1998a; Lambert et al. 1998). The remarkably rapid evolution of Sakurai's object, however, outpaces all other stars, and provides a unique opportunity to study stellar evolution during the very act, using all modern means of observations. We have monitored the evolution of Sakurai's object since discovery in 1996 using high-resolution optical spectra (Asplund et al. 1998b), which has allowed us to trace the rapidly changing appearance of the star both in terms of surface temperature and chemical composition.

\section{Observations and analysis}

Several studies of the chemical composition of Sakurai's object have been presented during the last years (Asplund et al. 1997b; Kipper \& Klochkova 1997; Shetrone \& Keane 1998). However, since the various analyses have relied on very different types of model atmospheres and have utilized different spectral features, it has been difficult to directly compare the results. Here we present a homogeneous re-analysis of all available published spectra of the star from 1996 with appropriate model atmospheres (Asplund et al. 1997a) and whenever possible the same spectral lines to avoid systematic differences.

The spectra have been obtained with various telescopes at varying resolution and S/N. The May and October 1996 spectra were acquired with McDonald Observatory 2.1 and $2.7 \mathrm{~m}$ telescopes at a resolving power of 30-60000 (Asplund et al. 1997b). Sakurai's object was also covered with the CTIO $4 \mathrm{~m}$ telescope in April 1996 (Shetrone \& Keane 1998) and with the SAO $6 \mathrm{~m}$ telescope in July 1996 (Kipper \& Klochkova 1997) though with slightly inferior resolution. The $\mathrm{S} / \mathrm{N}$ of the individual spectra varies between 75 and 200 . We defer the analysis of the 1997 and 1998 spectra to a later study, mainly due to the added complications arising from the terribly rich atomic and molecular line spectrum of later dates.

Especially constructed line-blanketed, H-deficient model atmospheres similar to those of Asplund et al. (1997a) formed the basis of the analysis. The stellar parameters were estimated using a range of ionization balance (mainly $\mathrm{N}$ I/N II, $\mathrm{Si}$ I/Si II, CrI/CrII, and FeI/FeII), excitation balance (OI, Fe I and Fe II) and line strengths of sensitive spectral features $\left(\mathrm{C}_{2}, \mathrm{C} I \mathrm{I}\right.$ and $\left.\mathrm{He} \mathrm{I}\right)$. Further information was provided by the hydrogen Balmer line profiles and the $(B-V)_{0}$ colours (Duerbeck et al. 1997). The microturbulence parameter was estimated from $\mathrm{CI}$, Ti II, Fe I and Fe II lines of various strengths. The spectroscopically derived $T_{\text {eff }}$ agrees very well with the photometric estimates (Duerbeck et al. 1997) using the same model atmospheres as those adopted in the present study, as evident from Fig. 1. Typical uncertainties are $\Delta T_{\text {eff }}= \pm 300 \mathrm{~K}, \Delta \log g= \pm 0.3 \mathrm{dex}$, $\Delta \xi_{\text {turb }}= \pm 1.0 \mathrm{~km} \mathrm{~s}^{-1}$. From the $\mathrm{C}_{\mathrm{II}}$ and He I lines in the April and May spectra, $\mathrm{C} / \mathrm{He}=10 \%$ (by number) was estimated. It should be noted, however, that the derived abundance from the $C_{1}$ lines result in a discrepancy of 0.6-0.7 dex: the same magnitude of the infamous "carbon problem" as those hampering all analyses of R CrB stars and related objects (Asplund et al. 1997a; Asplund et al. 1997b; Asplund et al. 1998a; Asplund et al. 1998b; Kipper \& Klochkova 1997; 
Table 1. The chemical compositions of Sakurai's object in 1996 (normalized to $\left.\log \Sigma \mu_{i} \epsilon_{i}=12.15\right)$. The errors quoted in the table are the formal statistical errors; no error is given in case the abundance is derived from a single line. Uncertain values are marked with ":"

\begin{tabular}{|c|c|c|c|c|c|c|}
\hline \multirow{2}{*}{ Element } & \multirow[t]{2}{*}{ Sun } & \multicolumn{4}{|c|}{ Sakurai's object in 1996} & \multirow{2}{*}{$\Delta \log \epsilon_{i}$} \\
\hline & & April 20-25 & May 5-9 & July 3 & October 7 & \\
\hline $\mathrm{H}$ & 12.00 & 10.0 & 9.7 & 9.6 & 9.0 & $\Downarrow 1.0$ \\
\hline $\mathrm{He}$ & 10.93 & 11.4 & 11.4 & 11.4 & 11.4 & 0.0 \\
\hline $\mathrm{Li}$ & 3.31 & 3.6 & 3.6 & 4.0 & 4.2 & $\Uparrow 0.6$ \\
\hline $\mathrm{C}$ & 8.52 & $9.7 \pm 0.2$ & $9.7 \pm 0.2$ & $9.7 \pm 0.3$ & $9.8 \pm 0.3$ & 0.1 \\
\hline $\mathrm{N}$ & 7.92 & $9.0 \pm 0.3$ & $8.9 \pm 0.4$ & $9.2 \pm 0.3$ & $8.9 \pm 0.2$ & 0.3 \\
\hline $\mathrm{O}$ & 8.83 & $9.2 \pm 0.2$ & $9.5 \pm 0.3$ & $9.3 \pm 0.1$ & $9.4 \pm 0.2$ & 0.3 \\
\hline $\mathrm{Ne}$ & 8.08 & $9.4 \pm 0.2$ & $9.4 \pm 0.3$ & $9.5 \pm 0.3$ & & 0.1 \\
\hline $\mathrm{Na}$ & 6.33 & $6.6 \pm 0.1$ & $6.7 \pm 0.1$ & $6.6 \pm 0.2$ & $6.8 \pm 0.1$ & 0.2 \\
\hline $\mathrm{Mg}$ & 7.58 & $6.5 \pm 0.4$ & $6.6 \pm 0.4$ & $6.3: \pm 0.4$ & $6.5 \pm 0.3$ & 0.3 \\
\hline $\mathrm{Al}$ & 6.47 & $6.5 \pm 0.2$ & $6.6 \pm 0.2$ & $6.6 \pm 0.3$ & 6.3 & 0.3 \\
\hline $\mathrm{Si}$ & 7.55 & $7.3 \pm 0.0$ & $7.1 \pm 0.2$ & $7.1 \pm 0.0$ & $7.5 \pm 0.2$ & 0.4 \\
\hline $\mathrm{P}$ & 5.45 & 6.2 & $6.2 \pm 0.4$ & 6.3 & & 0.1 \\
\hline $\mathrm{S}$ & 7.33 & $6.8 \pm 0.1$ & $6.6 \pm 0.1$ & $6.7 \pm 0.1$ & $6.9 \pm 0.1$ & 0.3 \\
\hline $\mathrm{K}$ & 5.12 & & $4.9 \pm 0.0$ & $5.0 \pm 0.1$ & $5.0 \pm 0.0$ & 0.1 \\
\hline $\mathrm{Ca}$ & 6.36 & $5.2 \pm 0.1$ & $5.6 \pm 0.3$ & $5.6 \pm 0.4$ & $5.5 \pm 0.4$ & 0.4 \\
\hline $\mathrm{Sc}$ & 3.17 & $3.1 \pm 0.1$ & $3.1 \pm 0.1$ & $3.3 \pm 0.2$ & $3.9 \pm 0.2$ & $\Uparrow 0.8$ \\
\hline $\mathrm{Ti}$ & 5.02 & $4.0 \pm 0.1$ & $4.1 \pm 0.2$ & $4.4 \pm 0.2$ & $4.6 \pm 0.2$ & $\Uparrow 0.6$ \\
\hline $\mathrm{Cr}$ & 5.67 & $4.5 \pm 0.1$ & $4.5 \pm 0.2$ & $4.8 \pm 0.2$ & $5.1 \pm 0.2$ & $\Uparrow 0.6$ \\
\hline $\mathrm{Fe}$ & 7.50 & $6.4 \pm 0.2$ & $6.4 \pm 0.2$ & $6.6 \pm 0.2$ & $6.6 \pm 0.3$ & 0.2 \\
\hline $\mathrm{Ni}$ & 6.25 & $6.1 \pm 0.3$ & $6.1 \pm 0.4$ & $6.0 \pm 0.2$ & $6.2 \pm 0.2$ & 0.2 \\
\hline $\mathrm{Cu}$ & 4.21 & & $5.0 \pm 0.3$ & $5.1 \pm 0.0$ & $5.0 \pm 0.1$ & 0.1 \\
\hline $\mathrm{Zn}$ & 4.60 & $4.9 \pm 0.2$ & $4.8 \pm 0.2$ & 5.1 & 5.4 & $\Uparrow 0.6$ \\
\hline $\mathrm{Rb}$ & 2.60 & & $<3.7$ & & 4.6 & $\Uparrow>0.9$ \\
\hline $\mathrm{Sr}$ & 2.97 & $4.7: \pm 0.1$ & $4.9: \pm 0.2$ & & $5.4: \pm 0.0$ & $\Uparrow 0.7$ \\
\hline Y & 2.24 & $3.2 \pm 0.3$ & $3.3 \pm 0.3$ & $3.7 \pm 0.2$ & $4.2 \pm 0.2$ & $\Uparrow 1.0$ \\
\hline $\mathrm{Zr}$ & 2.60 & $3.1 \pm 0.2$ & $3.0 \pm 0.3$ & $3.3 \pm 0.2$ & $3.5 \pm 0.3$ & $\Uparrow 0.5$ \\
\hline $\mathrm{Ba}$ & 2.13 & $1.5 \pm 0.1$ & $1.5 \pm 0.2$ & $1.8 \pm 0.1$ & $1.9 \pm 0.4$ & 0.4 \\
\hline $\mathrm{La}$ & 1.17 & & $<1.6$ & 1.3 & 1.5 & 0.2 \\
\hline
\end{tabular}

Lambert et al. 1998). The reason for this anomaly is still unknown but it may reflect inadequacies in the modelling of the stellar photospheres (Lambert et al. 1998). Fortunately, the derived abundance ratios such as $[\mathrm{X} / \mathrm{Fe}]$ are relatively immune to the carbon problem (Lambert et al. 1998) and therefore important conclusions regarding the evolution of Sakurai's object can still be drawn, in particular in light of the very extreme abundance ratios presented by Sakurai's object. 


\section{Elemental abundances}

The derived LTE abundances in Sakurai's object throughout 1996 are presented in Table 1. Two important conclusions are immediately obvious from an inspection of the table. First, the abundances of most elements (He, C, N, O, Ne, Na, $\mathrm{Mg}, \mathrm{Al}, \mathrm{Si}, \mathrm{P}, \mathrm{S}, \mathrm{K}, \mathrm{Ca}, \mathrm{Fe}, \mathrm{Ni}, \mathrm{Cu}$ and $\mathrm{La}$ ) have remained the same within the uncertainties (about $0.3 \mathrm{dex}$ ), which is reassuring and strengthens our confidence in the estimated stellar parameters. Needless to say, the stellar parameters were obtained before considering the abundances of individual elements, and no a posteriori modifications have been allowed. Second, several elements show abundance alterations which significantly exceed the statistical scatter and the estimated errors, and thus likely reflect real variations. The elements having undergone such alterations are $\mathrm{H}$ (decrease by $1.0 \mathrm{dex}), \mathrm{Li}(+0.6 \mathrm{dex}), \mathrm{Sc}$ $(+0.8 \mathrm{dex}), \mathrm{Zn}(+0.7 \mathrm{dex}), \mathrm{Rb}(>+0.9 \mathrm{dex})$ and $\mathrm{Y}(+1.0 \mathrm{dex})$, and probably also $\mathrm{Ti}(+0.6 \mathrm{dex}), \mathrm{Cr}(+0.6 \mathrm{dex}), \mathrm{Sr}(+0.7 \mathrm{dex})$ and $\mathrm{Zr}(+0.5 \mathrm{dex})$. There are also some indications that the heavy $s$-element $\mathrm{Ba}$ has increased to a smaller extent but this conclusion is less secure. As will be shown below, all but the variations for $\mathrm{Ti}$ and $\mathrm{Cr}$ can be naturally explained by the mixing and nucleosynthesis which has ensued due to the final He-shell flash.

Considering the drastic abundance alterations for some elements, one has first to investigate whether they indeed reflect real variations. It is important to realize that all changes will not disappear with any alternatively adopted stellar parameters. The required modifications of $T_{\text {eff }}(\approx 1000 \mathrm{~K})$ to annul most of the alterations would be in severe conflict with the various $T_{\mathrm{eff}}-\log g$ criteria. Furthermore, most of the elements showing an abundance trend are not more sensitive to the adopted stellar parameters than other elements. Hence different parameters would introduce other changes less easily explainable in the context of a final He-shell flash event. Also the fact that all elemental abundances in question show a definite trend with time supports the conclusion that the variations are real. We have also investigated the effects of hyperfine and isotope splitting, since many of the analysed lines are by necessity relatively strong and are able to dismiss those as causing the changes. Finally, we believe our assumption of LTE in the analysis is unlikely to severely affect our interpretation of the reality of the variations, since in most cases we are utilizing many different lines which should be little influenced by departures from LTE. Also it seems less likely that the relatively modest changes in the adopted stellar parameters can cause differential NLTE effects of $\sim 1.0$ dex.

\section{Changes in chemical composition and $T_{\text {eff }}$}

The abundance alterations of certain elements in 1996 can be naturally explained by the mixing and nucleosynthesis which was initiated in connection with the final He-shell flash. Most striking is the diminishing $\mathrm{H}$-content, which reflects the ingestion and burning of the $\mathrm{H}$-rich envelope following the development of a convection zone due to the He-shell flash. H-burning is now in fact likely the dominating nuclear energy source; thus we are witnessing the nuclear fuel of the star being consumed. The generated energy from H-burning not only provides the luminosity but has also caused the significant expansion of the star 


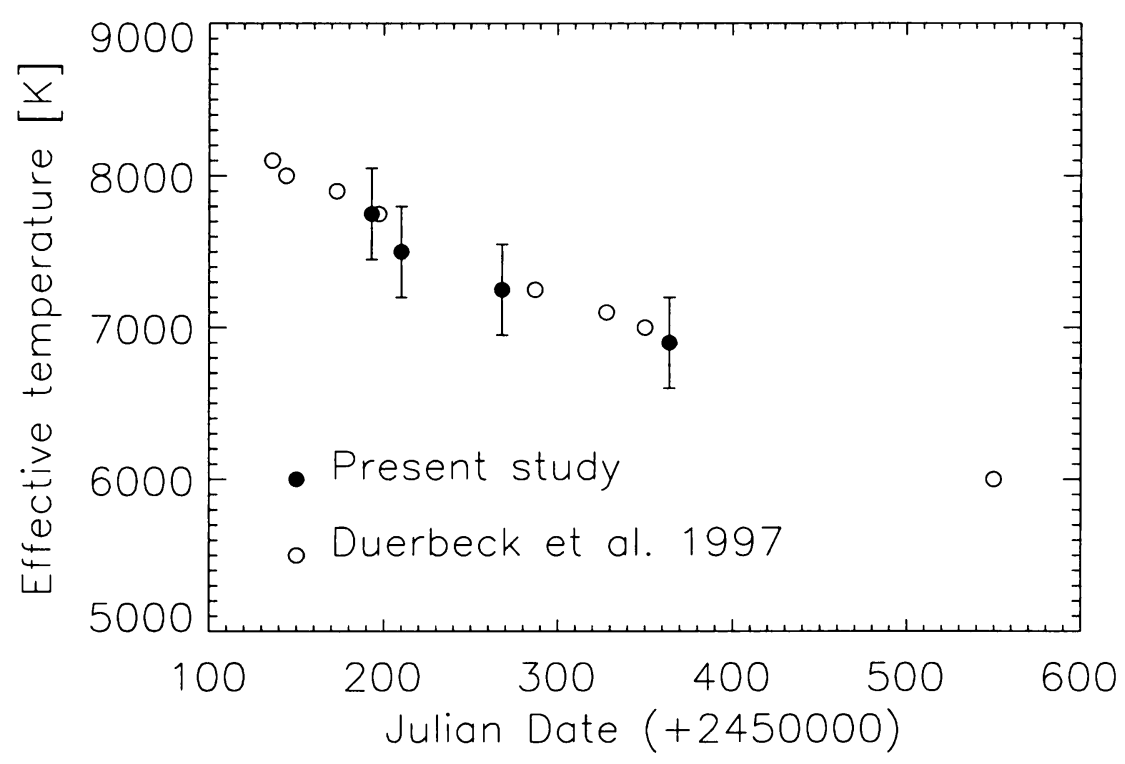

Figure 1. The evolution of the effective temperature of Sakurai's object as judged from spectroscopy (present study) and UBVRiz photometry (Duerbeck et al. 1997)

to giant dimensions. This expansion has resulted in a continued cooling of the stellar surface, as evident both from spectroscopy and photometry (Fig. 1); the cooling has continued throughout 1997 and 1998. The time-scale for the observed variations in $T_{\text {eff }}$ and chemical composition ( $\sim$ months) is significantly faster than predictions from the only published numerical calculation of such an event (Iben \& MacDonald 1995) but it can likely be accounted for with a slightly larger stellar mass (say $M_{*} \approx 0.8 \mathrm{M}_{\odot}$ instead of $M_{*}=0.6 \mathrm{M}_{\odot}$ ).

Further support for a second, current stage of CNO-cycling comes from the low observed ${ }^{12} \mathrm{C} /{ }^{13} \mathrm{C}(\approx 3)$; following He-burning, which is required to explain the over-abundances of $\mathrm{C}, \mathrm{N}, \mathrm{O}$ and $\mathrm{Ne}$, the ratio should be very high. If the proton supply is exhausted before completion, CNO-cycling will produce vast amounts of ${ }^{13} \mathrm{C}$, which can be brought down to He-burning temperatures and there release free neutrons. Sakurai's object shows evidence of very prominent $s$-processing, in particular of the light $s$-elements (including $\mathrm{Sc}, \mathrm{Ni}, \mathrm{Cu}$ and $\mathrm{Zn})$. The abundance distribution of the $s$-elements is best explained by a single neutron exposure, which suggests that the $s$-processing occurred during the final He-shell flash. However, the alternative interpretation that the $s$-elements stem from thermal pulses during the previous AGB-phase and now are being mixed to the surface can not be convincingly ruled out either. It should be noted that the seemingly simultaneous productions of ${ }^{13} \mathrm{C}$ and $s$-elements pose a serious challenge to theoretical modelling if correct, in particular how the ${ }^{13} \mathrm{C}$ is brought 
down to the He-burning environment, since the initiated H-burning should cause the convection zone due to He-burning to split into two.

$\mathrm{Li}$ is most easily explained by production through the Cameron-Fowler mechanism from ingested ${ }^{3} \mathrm{He}$ in the $\mathrm{H}$-rich envelope. Since $\mathrm{H}$-burning necessarily destroys $\mathrm{Li}$, the observed $\mathrm{Li}$ can not have been inherited from a previous AGB-phase but must be currently synthesized. The increases in $\mathrm{Ti}$ and $\mathrm{Cr}$ abundances, however, presently lack a nucleosynthetic explanation.

Sakurai's object has thus evolved significantly both in terms of surface temperature and in chemical composition within only six months. To our knowledge, Sakurai's object represents the fastest case of stellar evolution ever observed, when excluding complete stellar disruptions such as supernovae and novae.

\section{Similarities with the $\mathrm{R}$ CrB stars}

Sakurai's object shows striking similarities with the R CrB stars in terms of chemical composition. The $\mathrm{R} \mathrm{CrB}$ stars are distinguished by their prominent $\mathrm{H}$ deficiency and C-enrichment. Sakurai's object not only shares these properties, but also closely resemble the $\mathrm{R} \mathrm{CrB}$ stars in the observed $[\mathrm{X} / \mathrm{Fe}]$ ratios, though it has more pronounced over-abundances of the light $s$-elements and have a low ${ }^{12} \mathrm{C} /{ }^{13} \mathrm{C}$ ratio. Furthermore, Sakurai's object has recently been reported to experience the typical irregular visual variability of the $\mathrm{R} C r B$ stars $(\mathrm{H}$. Duerbeck and E. Guinan, private communication). Thus, Sakurai's object has evolved into being an R CrB star, which is the first time ever this has been observed. Sakurai's object therefore lends strong support to the suggestion that final He-shell flashes in pre-white dwarfs can produce these stars. It is, however, likely premature to conclude that all $\mathrm{R} \mathrm{CrB}$ stars have been formed similarly.

Acknowledgments. The author greatly appreciates the rewarding collaborations with B. Gustafsson, K. Jonsell, T. Kipper, D.L. Lambert, N.K. Rao, M. Shetrone and B. Smalley on the topic of Sakurai's object and related stars. Photometric data from H. Duerbeck and E. Guinan is acknowledged.

\section{References}

Asplund M., Gustafsson B., Kiselman D., Eriksson K., 1997a, A\&A 318, 521 Asplund M., Gustafsson B., Lambert D.L., Rao N.K., 1997b, A\&A 321, L17 Asplund M., Gustafsson B., Rao N.K., Lambert D.L., 1998a, A\&A 332, 651 Asplund M., Lambert D.L., Kipper T., Shetrone M.D., 1998b, A\&A, submitted Duerbeck H.W., Benetti S., Gautschy A., et al., 1997, AJ 114, 1657

Iben I., Jr., MacDonald J., 1995, in White dwarfs, D. Koester and K. Werner (eds.), Springer, Berlin, p. 48

Kipper T., Klochkova V.G., 1997, A\&A 324, L65

Lambert D.L., Rao N.K., Gustafsson B., Asplund M., 1998, A\&A, submitted Shetrone M.D., Keane M., 1998, in Faint blue stars, A.G. Davis-Philip (ed.), L.

Davis Press, Schenectady, in press 